\title{
Realization of Intelligent Grasping on a Traditional Hydraulic Manipula- tor
}

\author{
Shang Zhendong ${ }^{*}, 1$, Zhang Xiaolan ${ }^{2}$ and Zhou Yuting ${ }^{1}$ \\ ${ }^{I}$ School of Mechatronics Engineering, Henan University of Science and Technology, Luoyang, Henan, 471003, P.R. \\ China \\ ${ }^{2}$ School of Medical Technology and Engineering, Henan University of Science and Technology, Luoyang, Henan, \\ 471003, P.R. China
}

\begin{abstract}
In the literature, studies on dexterous manipulators are mainly focused on multi-finger multi-joint structures. These manipulators often have difficulties with grasping heavy objects due to the delicateness and complexity of the structures. This paper describes the authors' attempt on implementing dexterous grasping on a traditional two-single-jointfinger hydraulic manipulator. Hydraulic driven manipulators have higher power output. However, dexterous grasping realization on this type of manipulators represents a very high technical challenge as it is difficult to measure the changes in the grasping force and the object attitude. In the authors' study, an angle sensor and a force sensor are used to capture the changes in the finger opening distance and the grasping force. An elastomer and photoelectric device based slip sensor is designed to measure the relative movement between the fingers and the object. A fuzzy control algorithm is developed and implemented on a microcontroller unit to serve as the higher-level controller of a two-level hierarchical control system for the finger movement. The algorithm decides the feeds of the fingers based on the outputs of the angle, force and slip sensors. The lower-level controller is an electrical grasping motion control device that applies the output of the fuzzy controller to control the movement of the fingers. With a 10Mpa hydraulic power rate, the maximum grasping force of the manipulator is over $2500 \mathrm{~N}$. Experiments show that this hydraulic dexterous manipulator can grasp both heavy objects and eggs, glasses and other fragile objects.
\end{abstract}

Keywords: Dexterous grasping, fuzzy control, hierarchical control, hydraulic manipulator, slip sensor.

\section{INTRODUCTION}

The majority of the studies on dexterous manipulators reported in the literature have been focused on multi-finger multi-joint structures. C. V. Baysal et al. 2010 [1] proposed an intelligent inference system for multi finger manipulators. Y. K. Cho et al. 2011 [2] studied the control of a four finger manipulator. C. H. Chen et al. 2013 [3] investigated the hybrid control strategies for a five finger robotic hand. V. Lippiello et al. 2013[4] analyzed the dynamic properties of multi-finger manipulator. These manipulators were reported to have problems with grasping heavyweight objects because of the delicate and complex finger structures.

Hydraulic driven manipulators are proven to be more efficient in handling heavyweight objects and providing a larger operation range $[5,6]$. Many service robots and industrial robots use this type of manipulators. However, due to the high output force, it is very difficult to measure the precise change of the grasping force on this type of manipulators during operation. Therefore implementing dexterous grasping on hydraulic driven manipulators represents a high technical challenge.

*Address correspondence to this author at the School of Mechatronics Engineering, Henan University of Science and Technology, Luoyang, Henan, 471003, P.R. China; Tel: +8637964231479; Fax: +8637964231763; E-mail: cnzzszd@163.com
Some of the dexterous manipulators are designed for objects with known shapes only, while more manipulators are with object recognition capability. For example, [7] used the Levenberg-Marquardt algorithm in a monocular vision system to recognize the six-dimensional attitude of the objects in their intelligent grasp realization. W. H. Li et al. 2011 [8] realized object recognition by identifying bilateral symmetry of the object via machine vision. These approaches require complicated algorithms and control logic therefore often lead to high cost.

The authors of this paper investigated realization of dexterous grasping on a conventional two-single-joint-finger hydraulic driven manipulator. In this system, some simple sensors are used to measure the dynamic changes of the object and the finger. These measurements are then fed into a two-level hierarchical control system, which includes a fuzzy controller, to control the operation of the fingers. Experiments show that the manipulator is capable to carry out dexterous grasping on a wide range of objects and therefore prove that this is a low cost and high efficiency approach.

\section{SYSTEM OVERVIEW AND OPERATION PRINCI- PLE}

\subsection{Manipulator Structure}

In this study, a conventional hydraulic driven manipulator was modified to dexterous grasping. The mechanical 


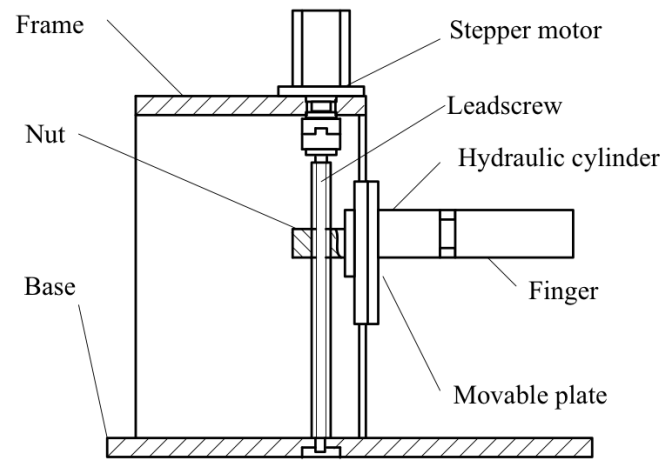

(a) Overall structure

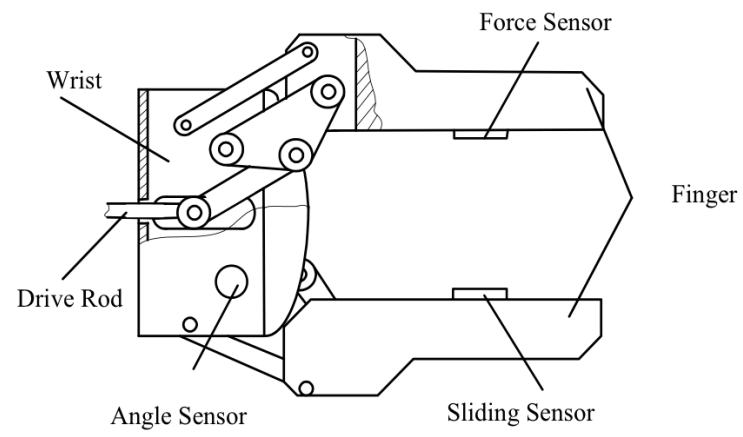

(b) Finger structure

Fig. (1). The mechanical structures of the manipulator.

structure of the manipulator is shown in Fig. (1a). The structure shown in Fig. (1b) is just for a single finger as the two fingers of the manipulator are exactly the same. The general architecture of the manipulator is shown Fig. (2). It comprises of the frame, mechanical finger, the lifting mechanism, a hydraulic subsystem and the control subsystem. The lifting mechanism comprises a stepper motor, a lead screw and nut mechanism and a lifting board. The finger is a typical mechanical linkage, which turns the linear movement of the piston into opening and closing actions. A microcontroller based control unit is the main body of the control system. It provides control signals to the electrical control circuits to drive the stepper motor of the lifting mechanism and the servo valves that controls finger movement.

\subsection{Operation Principle}

Dexterous grasping requires servo control on the finger feed amount and the grasping force. The mechanical hydraulic manipulator often outputs a very large force. This brings great difficulties to the design of the control policy unit as dexterous grasping requires very fine control on the grasping force change. In addition, owing to the inherent nonlinearity of hydraulic systems as well as complicated load variations, it is very difficult to achieve ideal control precision with traditional proportional-integral-derivative (PID) control.
Therefore, fuzzy logic based control is considered as an optimal solution. In this study, a two-level hierarchical control system was developed. Its upper level is a fuzzy controller running on a microcontroller unit (MCU) that provides the finger feed amount control. The lower level is an electrical control circuit that implements the feed amount from the fuzzy controller to control directly the finger actions.

When the system starts grasping, the control system drives the gripper to close and stop the closing action when the force sensor detects the object being contacted by the finger. The slip detection is then started. The fuzzy control system takes the measurements from the slip sensor and the force sensor to work out fuzzy decisions to control the finger movement via the low-level control circuit. When the object is firmly clamped and no slip is detected, the control system drives the stepper motor to lift the object.

\section{SENSORS}

In this study, we added an angle sensor, a force sensor and a slip senor to the fingers of the manipulator. Fig. (1b) illustrates where these sensors are installed. The angle sensor is fixed on one of the hinge axis of the linkage that drives the finger opening and closing movement, to measure the rotation angle of the hinge axis which can then be converted into 


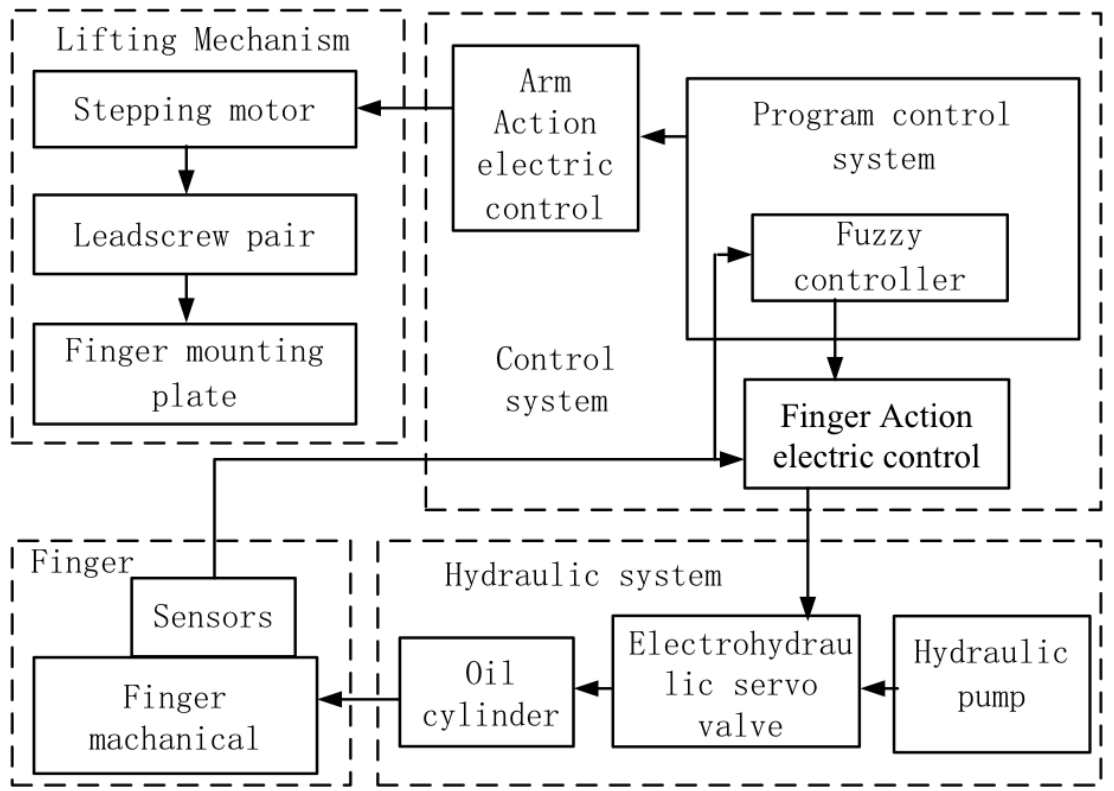

Fig. (2). Architecture of the modified hydraulic-driven manipulator.

the opening distance of fingers by calculation. The force sensor is fixed on a finger to determine the holding force on the object. The slip sensor is fixed on another finger. It includes an elastomer that responds the slip intention of the object against the fingers and a photoelectric circuit that converts the bending deformation of the elastomer to an electrical signal that represents the magnitude of the slip intention.

\subsection{Angle Sensor}

The angle sensor installed in the manipulator (see Fig. 1) measures the opening distance between the two fingers. The sensor is basically a rotation transformer and is mounted on a rotation axis of the manipulator to measure the rotation angle of the axis so that the distance between the two fingers can be obtained through further calculations.

The finger structure in Fig. (1) can be simplified as a linkage as shown in Fig. (3). In the diagram, $A B C$ is a rigid link rod. A is the hinge. D is the moving end of the piston which moves in the vertical direction reciprocatively and therefore drives $\mathrm{ABC}$ via the link rod $\mathrm{BD}$ to realize the clamping and releasing of the fingers. The horizontal displacement of point $\mathrm{C}, \mathrm{d} k$, is the opening distance of the finger.

In the Fig. (3), $\mathrm{ABC}$ is the initial position of the finger. The dashed line shows a position during finger opening. The forward distance of $\mathrm{D}$ is denoted as $\mathrm{d} f$ and the rotation angle at Point $\mathrm{A}$ as $\theta$. Since $\angle D A B=\theta_{1}, \angle D A X=\alpha_{1} \angle D^{\prime} A B^{\prime}=\theta_{2}$ and $\angle D^{\prime} A X=\alpha_{2}, \angle B A B^{\prime}$ is the rotation angle $\theta$.

From the known conditions, we can get:

$\cos \theta_{1}=\frac{A B^{2}+A D^{2}+B D^{2}}{2 A B \cdot A D}$

$\theta_{1}=98.63^{\circ}$

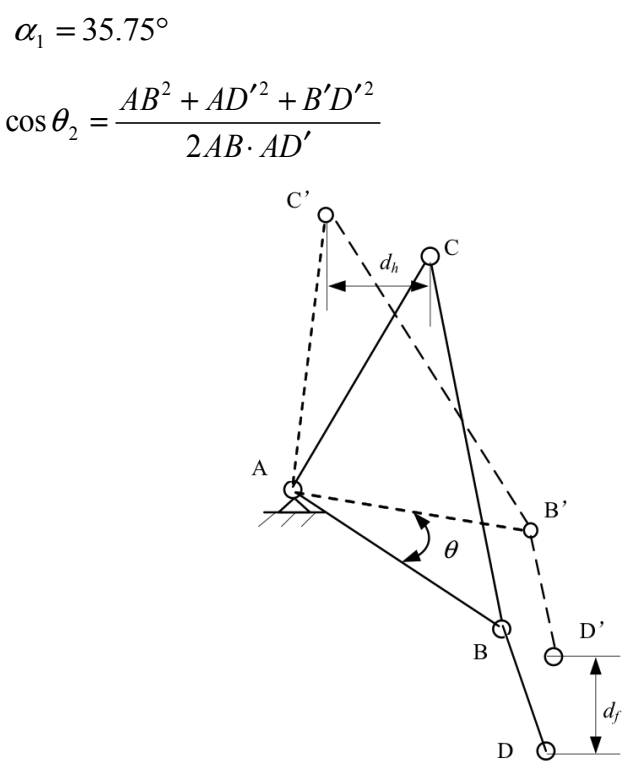

(a) Motion analysis

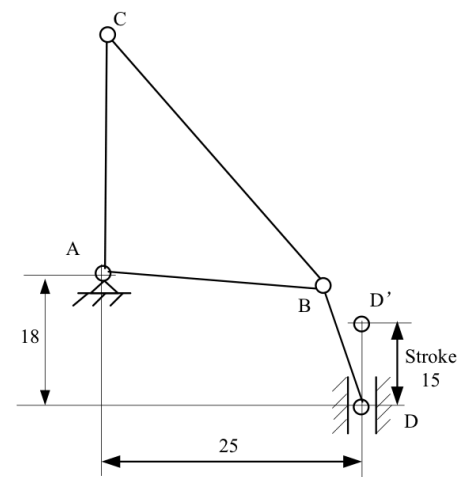

(b) Structure size

Fig. (3). Sketch of the finger motion and size. 
Substituting the relative parameters into the equation, then we get:

$\cos \theta_{2}=\frac{\left(18-d_{f}\right)^{2}-620}{64 \sqrt{625+\left(18-d_{f}\right)^{2}}}$

$\tan \alpha_{2}=\frac{18-d_{f}}{25}$

The rotation angle $\theta$ of point $\mathrm{A}$ can be calculated by the equation below:

$\theta=\arccos \frac{\left(18-d_{h}\right)^{2}-620}{64 \cdot \sqrt{625+\left(18-d_{h}\right)^{2}}}$

$-\arctan \frac{18-d_{h}}{25}-62.37 \times \frac{\pi}{180}$

The relationship between the horizontal displacements $d_{h}$ of the fingers and the rotation angle $\theta$ is:

$d_{h}=A C^{\prime} \cdot \sin \theta$

According to the structure parameters of fingers, AC is $60 \mathrm{~mm}$ and $\theta$ is between $0 \sim 20^{\circ}$. Thus the maximum opening distance of the finger is around $81 \mathrm{~mm}$. Moreover, the relationship between the piston position in the cylinder and the rotation angle is as bellow:

$d_{h}=60 \sin \theta$

\subsection{Force Sensor}

In this system, a force sensor is mounted on one of the fingers of the manipulator to measure the clamping force applied on the object, i.e., the interactive force between the gripper and the object. The precision requirement on the actual measurement is not high. This is because the force is a fuzzy value, which is of relative (not absolute) significance, during the soft grasping process. The strength of the force required for holding up an object cannot be determined in advance. It is the slippage of the object that is of absolute significance. However, force detection is essential to the system. The force sensor is mainly responsible for detecting if the object is contacted by the gripper and provides the detected force strength to the fuzzy controller as a reference for determining the finger's feed required for gripping the object. Therefore, a linear relation between the gripping force and the gripper displacement is more important.

Here, we designed a simple and practical force sensor based strain gauges. The sensor is shaped as a thin rectangular prism. Its top side directly contacts with the object and is covered with a thin layer of rubber to improve the surface's friction and elasticity. Four pieces of strain gauges are attached on the opposite side of the sensor to form an electrical conductance measuring bridge, where the horizontally positioned two strain gauges are used for detecting the micro deformation and the vertically positioned two are used for temperature compensation. The sensor is mounted on the manipulator through the tap holes. When there is a pressure on the top surface of the sensor, the horizontally positioned strain gauges become deformed as the result of stretch. This leads to the gauge's resistance changes, which can be converted into voltage changes by the associated electronic circuit. The vertically placed strain gauges are not sensitive to the stretch. It can be regarded that their resistances remain unchanged during the operation.

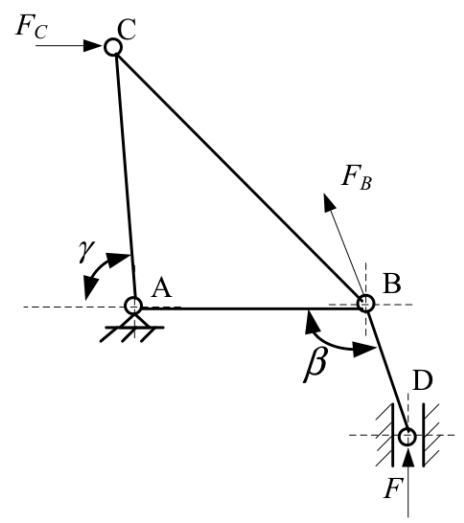

Fig. (4). Stress analysis of the gripper.

In Fig. (4), $F$ is the thrust of the cylinder piston is, $F_{B}$ is the thrust on the $\mathrm{ABC}$ shape from the DB rod and $F_{c}$ is the horizontal force on point $\mathrm{C}$, i.e. the clamping force of the gripper. Through a moment balance analysis for the $\mathrm{ABC}$, we have the following equations:

$F_{B} \cdot A B \cdot \sin \beta=F_{C} \cdot A C \cdot \sin \gamma$

$F_{B}=\frac{F}{\cos \alpha}$

Combining (5) and (6), we can get:

$F_{C}=\frac{F \cdot A B \cdot \sin \beta}{A C \cdot \sin \gamma \cdot \cos \alpha}$

When grasping an object, $F_{c}$ is the positive pressure between the gripper and the object. When a rated output of $10 \mathrm{MPa}$ is selected for the hydraulic power unit, the maximum grasping force is $2533 \mathrm{~N}$.

It should be noted that the direct feedback into the fuzzy controller is the position signal, not the force signal. This requires the relationship between the gripper position change and the force change to be revealed. An experiment-based study was carried out for this purpose. In the experiments, we used the manipulator to grasp rigid objects. Form the point that the gripper contacts the object, an incremental feed value was given to the gripper and the corresponding force strength values from the force sensor were recorded. The result of the experiments is shown in Fig. (5). From the diagram, we can observe that a relationship between the feed of gripper and the detected clamping force is non-linear. This non-linearity is caused by the manufacturing techniques and 
the rubber layer on the sensor surface. To realize the force control stated above, linearization for the curve in Fig. (5) is required. We divided the curve in Fig. (5), into three sections. Each of them was approximated into a straight line with a different slope. Each line represents a different working state for the force sensor. As will be described later, the fuzzy controller picks up different parameters for the different working state of the force sensor to make control decisions.

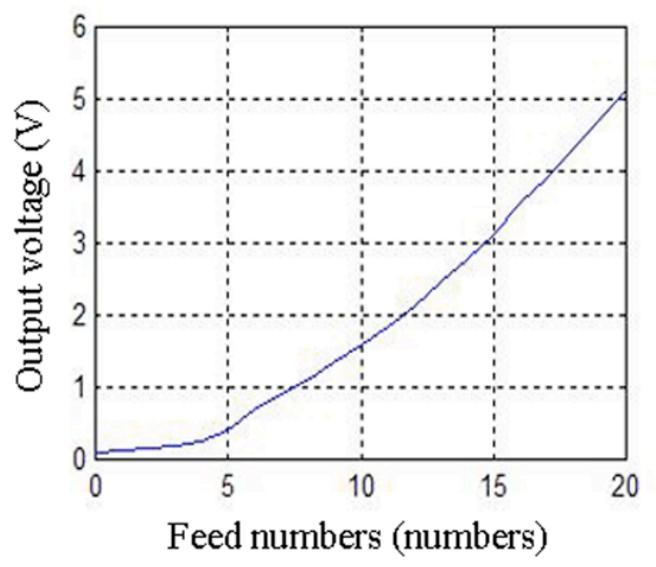

Fig. (5). Mapping of the feed number and the output voltage.

\subsection{Slip Sensor}

The other finger of manipulator is mounted with the slip sensor to measure the slippage of the object against the finger. The structure of slip sensor and its installation method is shown in Fig. (6). The base of the sensor is fixed onto the finger directly and the elastomer is mounted on the base through screw thread. The elastomer is of a cylinder shape with transverse grooves so that its surface contacts have the level of elasticity in both axial and radial directions required for sensing the movement of the object.

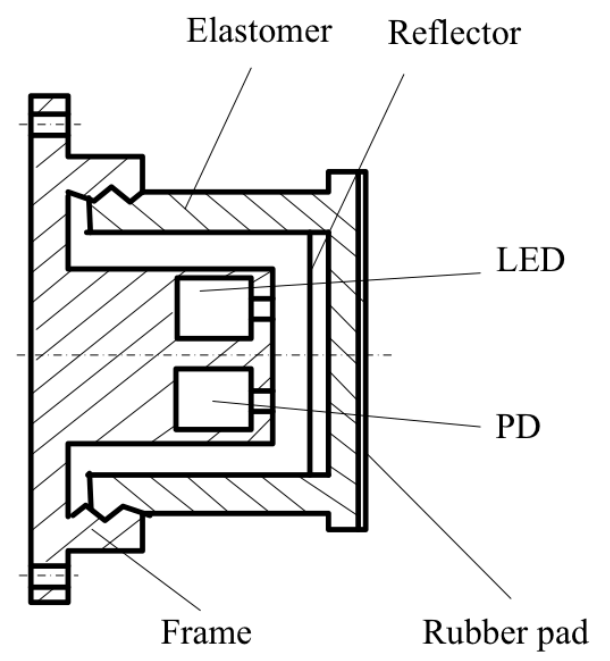

Fig. (6). Sketch map of slipping sensor's structure.

Fig. (6) illustrates the operation principle of the slip sensor. The light emitting diode (LED), photoelectric device (PD) and their driving and signal processing circuits are encapsulated in the chamber of the slip sensor's base. The reflecting mirror (reflector) is installed on the other side of the chamber. The light from the LED travels through a hole to reach the reflector and then reflected back to the surface of the photodiode device via another hole. When the object slips against the finger of manipulator, the friction between the object and the finger drives the contact end of the elastomer to move downwards. However, the elastic force of the elastomer prevents such movement. Hence, these forces cause micro-vibration at the contact end of the elastomer, which drives the reflector to make the same movement. This motion causes the angle of the mirror surface to change constantly, hence leads to a constant change in the reflection angle of the incident light against the mirror surface. This change is reflected on the luminous flux received by the photodetector and thus on the voltage output of the photo detection circuit.

The slippage of the object is positively correlated with the differential of the output voltage of the circuit $(\mathrm{d} u(t) / \mathrm{d} t)$ and is negatively correlated with the mass of object $\left(m_{\mathrm{o}}\right)[9]$. Meanwhile, these correlations are linear. The slippage has a certain non-linear correlation with the voltage output value $u(t)$ itself. However, since the deflection at the elastomer contact caused by the micro-vibration is very small, this correlation can be ignored.

The following experiment was conducted for testify the effectiveness of the slip sensor. The gripper grasped an object under hand-operated control and then the stepper motor was driven by the microcontroller to lift the gripper up for a short distance at a constant speed. The output signal of the slip sensor was recorded by a personal computer (PC) into a data file simultaneously. In the experiment, the following test cases were carried out so as to testify the effectiveness of the slip sensor: Varying the gripping force for a same object to observe the detection effect for different gripping force conditions; Applying a same gripping force to different objects to observe the detection effect for different surface conditions.

Fig. (7) shows the experiment result for a glass cup grasping test, where the gripping force was gradually increased during the grasping operation.

In the Fig. (7a) shows the experimental curve for the initial contact stage where the gripping force is small, (b) shows the experimental curve for the intermediate stage where the gripping force is being increased but not large enough to clamp the cup and (c) shows the curve for the clamped stage. From the curves, we can draw a conclusion that the slip sensor can effectively detect the slippage of the object:

From the Fig. (7a) and (b), it can be seen that, for the same object, the amplitude of slip sensor output signal becomes larger when the force is larger. This is because that, when the gripping force is small, the small friction between the object and the sensor contact surface caused by the slippage results in a low amplitude vibration in the sensor contact. When the grasping force becomes larger, the friction between object and the sensor surface increases. This leads to an increase in the amplitude of the vibration, which 
reflected in the sensor's voltage output. For the similar reason, when the same gripping force is applied, the slip sensor output is small for an object with a smooth surface and is larger for an object with a rough surface. The slip sensor output cannot increase further when the gripping force reaches the maximum value but the object is not slipping. It can be seen from (c) that when the object does not slip, the output of the slip sensor maintains at a certain initial value and fluctuates with the range of $\pm 0.02 \mathrm{~V}$. This fluctuation can be easily filtered out in software.

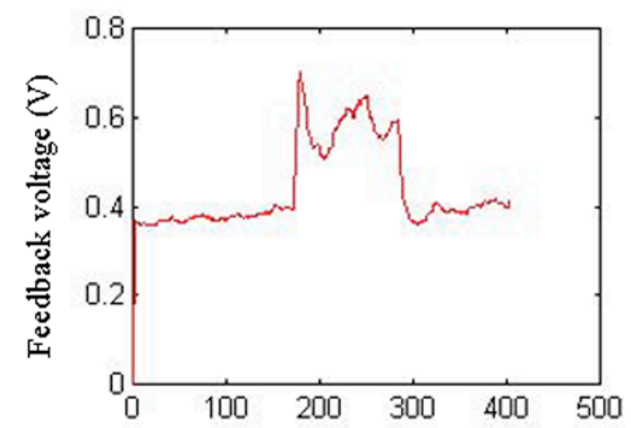

Numbers of sampling points (numbers)

(a) The curve for the initial contact stage

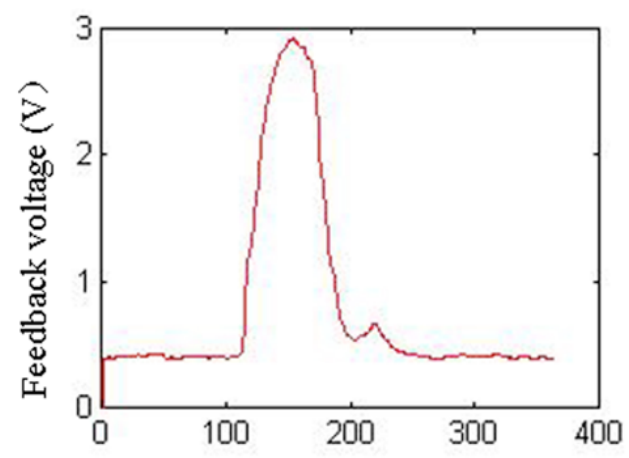

Numbers of sampling poings (numbers)

(b) The curve for the intermediate stage

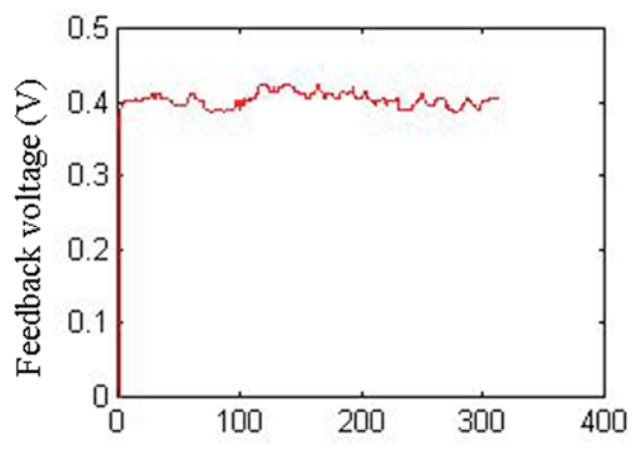

Numbers of sampling points (numbers)

(c) The curve for the clamped stage

Fig. (7). Experimental characteristics of the slip sensor.

\section{FINGER ACTION CONTROL SYSTEM}

In our study, a two-level hierarchical control system based on a microcontroller is implemented to control the operation of the manipulator. Fig. (2) shows the block diagram for the finger action control subsystem. The upper level of the system is a fuzzy controller that takes the output of the force and slips sensors as feedback signals and decides the grasping force of the fingers. The lower level is a finger action electrical controller, which is a conventional electrical control circuit and implements the decisions from the fuzzy controller to drive the hydraulic servo system to control the fingers' actions.

\subsection{Finger Action Electrical Controller}

As shown in Fig. (2), the finger action control system composes a hydraulic power source, a servo valve, a line oil cylinder, an angular displacement transducer and a finger action electrical controller. According to our study, the open loop transfer function of the system $W_{K}(s)$ :

$$
W_{K}(s)=\frac{g}{s \cdot\left(1+a_{1} s\right)\left(a_{2} s^{2}+a_{3} s+1\right)}
$$

Where $g$ is the total system gain and $a_{1}, a_{2}$ and $a_{3}$ are the parameters of the system inertial and oscillation elements. It can be seen from (8) that the model for the system is a fourstep system including an integral segment, an inertia segment and an oscillation segment.

We used a closed loop finger action control experiment to verify the above open loop transfer function. It is known from simulation that the system does not have a good performance and is in a critical steady state and has a high shock. A compensator must be added into the system. Based on some experiments and theoretical analysis, we set the following design specifications for the compensation: the system tracing error is $1 \%$, the overshoot is no more than $5 \%$ and the system delay is as small as possible. A lead-lag compensator is chosen to implementation this. The system transfer function of the designed compensator is:

$$
G_{c}(s)=\frac{(0.25 s+1)(0.2 s+1)}{(2.5 S+1)(0.67 S+1)}
$$

The characteristic curves of amplitude-frequency and phase-frequency of the system after compensation are shown in Fig. (8). The crossover frequency $\left(W_{x}\right)=26.8 \mathrm{rad} / \mathrm{s}$, the phase margin $\left(P_{m}\right)=65.3^{\circ}$, the gain margin $\left(G_{m}\right)=3.7 \mathrm{~dB}$. It satisfies the design requirements for the controller.

\subsection{Fuzzy Control System}

The top level of the control system is a fuzzy strategy based humanoid decision making controller. As already shown in the dotted box of Fig. (2), the fuzzy controller is implemented as an embedded software application running on the MCU. The application comprises the following four 

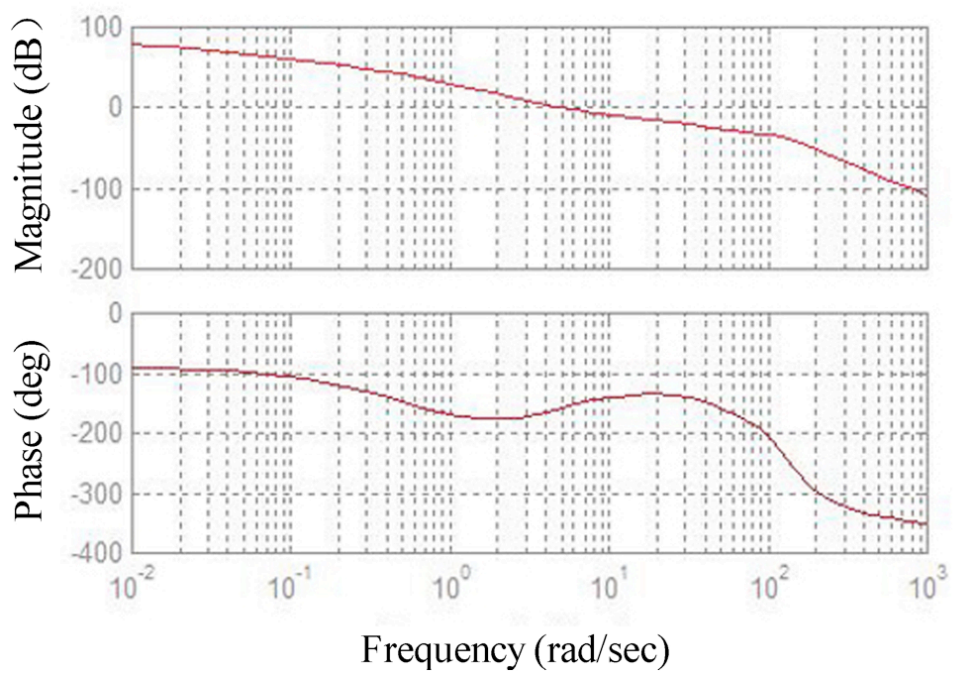

Fig. (8). Amplitude-frequency and phase-frequency characteristics after compensation.

Table 1. Fuzzy control rule table.

\begin{tabular}{|c|c|}
\hline No. & Fuzzy Control Rules \\
\hline 3 & IF $S=\mathrm{PB}$ AND $S C=\mathrm{PS}$ THEN $U=\mathrm{PM}$ \\
\hline 4 & IF $S=$ PS AND $S C=$ PB THEN $U=\mathrm{PM}$ \\
\hline 6 & IF $S=\mathrm{PB}$ AND $S C=\mathrm{PB}$ THEN $U=\mathrm{PB}$ \\
\hline 7 & IF $S=$ PS AND $S C=$ PM THEN $U=$ PS \\
\hline 8 & IF $S=\mathrm{PM}$ AND $S C=\mathrm{PM}$ THEN $U=\mathrm{PM}$ \\
\hline 9 & IF $S=$ PB AND $S C=$ PM THEN $U=\mathrm{PM}$ \\
\hline
\end{tabular}

parts: a fuzzification module, a knowledge base, a fuzzy inference engine and a defuzzification module.

Fuzzy control is to eliminate the slippage error of the object (the difference between the measured and expected slippage). The slippage error signal $S$ and the slippage signal SC are the inputs. The control volume $U$ is the output. The fuzzy language descriptions (fuzzy sets) of $S, S C$ and $U$ are defined as follows: $S, S C$ and $U$ have the common fuzzy set $\{\mathrm{PS}, \mathrm{PM}, \mathrm{PB}\}$. PS, PM and PB represent the three levels of fuzzy values respectively: Positive Small, Positive Medium, and Positive Big. Both the domains of $S$ and $S C$ are the same: $\{1,2,3,4,5,6\}$. The domain of $U$ is $\{1,2,3,4,5,6,7\}$.

As described earlier, the system has different control error regions. Different fuzzy subsets are selected for the different regions. A fuzzy control system's sensitivity is determined by the resolution of the fuzzy set. With a higher resolution, the system has higher control sensitivity. And conversely, with a lower resolution, the system has a slower control characteristic but a better stability. Therefore, a fuzzy set with a lower resolution should be adopted in the region with a larger error and a fuzzy set with a higher resolution should be adopted in the zone with a smaller error.

A fuzzy set's resolution is reflected on the shape of its membership function curve. The sharper the curve is, the higher the resolution is. In this study, fuzzy sets with normal style membership functions were selected through experiments and simulations. The assignment tables of the fuzzy variables $S, S C$ and $U$ are obtained from experiments.

The establishment of the fuzzy control rules was based on our experiments. Fuzzy reasoning can be conducted based on the above established fuzzy relations:

$U^{\prime}=\left(S^{\prime} \cup S C^{\prime}\right)_{\circ} \quad R$

Where: the symbol "o" indicates a synthesis operation, where a maximization-minimization method is used. The control value $U^{\prime}$ can be obtained via reasoning the input $S^{\prime}$ and $S C^{\prime}$.

The nine control rules are listed in Table 1 . The procedure can be applied to the rest of the rules in the table, i.e. 


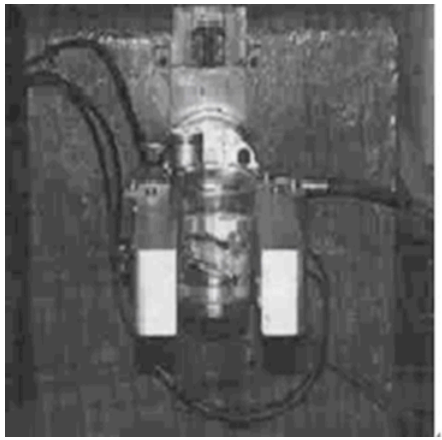

(a)

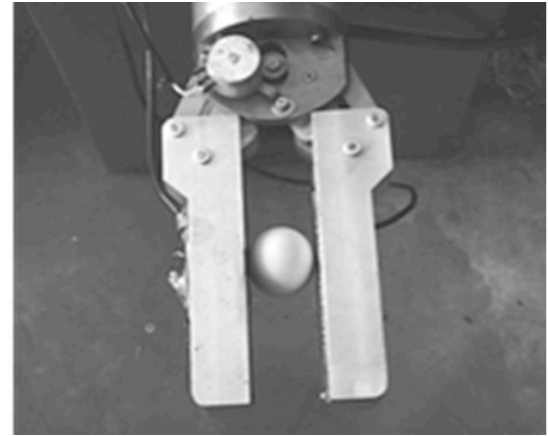

(b)

Fig. (9). The grasping of fragile objects such as glasses, eggs without damage.

$\mathrm{R} 1 \sim \mathrm{R} 9$, to obtain the fuzzy relation $R$. The fuzzy inference can be performed using Formula (10) based on the obtained fuzzy relation.

To implement precise control, the fuzzy quantity needs to be converted into an accurate quantity, i.e. defuzzified. The accurate quantity is a digital value and is converted by a digital-to-analogue converter (ADC) of the MCU into an analog signal, which is the control input of the finger action electrical controller. During the gripping operation, the continuous feedback signals from the sensors are sampled by the ADC and fed into the fuzzy controller. A closed-loop fuzzy control on the object grasping is thus realized.

As all the above fuzzy sets have discrete domains, the number of the quantized input and output values is limited. This enables us to consolidate the input conditions and the corresponding control output volumes into a look-up table at off-line time so that the fuzzy calculation in the software during the real-time control operation is simplified as a table lookup operation. We used Formula (10) to obtain the output fuzzy set $U$ for various value combinations of the $S$ and $S C$ inputs and then used a weighted average calculation on $U$ for defuzzification to get the control output $u$.

The experiments shown as Fig. (9) demonstrate that, with the fuzzy controller described above, the two-single-jointfinger hydraulic manipulator can successfully grasp eggs and glass without damage.

\section{CONCLUSION}

This paper has described a novel approach for implementing dexterous grasping on hydraulic driven manipulators to avoid the heavy-load problem with multifigure/multi-joint dexterous grasping manipulators. In this approach, some simple sensors are used to measure the grasping process's dynamic parameters (e.g. the slippage, the holding force). These measurements are fed into a two-level hierarchical control system, which comprises a fuzzy logic based humanoid decision-making control algorithm that provides fine control over the finger actions. This approach enables the realization of intelligent grasping on a simple mechanical structure without using complicated object shape and gesture recognition elements. Experiments have shown that after modification, a conventional hydraulic driven manipulator with two single-joint fingers is capable with intelligent grasping and has all the necessary flexibility and lifting power: with a $10 \mathrm{MPa}$ rated hydraulic power, the maximum grasping force is above $2500 \mathrm{~N}$.

The slip sensor presented in this paper uses an elastomer and photoelectric devices to measure the object slippage against the manipulator fingers. Simulation and experiments show that the sensor has a high sensitivity and a good antiinterference capability.

For future work, the method proposed in this paper can be further improved. Firstly, the slip sensor's dependency on the shape of object can be further reduced by adopting a multiple-sensor strategy. Secondly, further study on the smart grasping control scheme can be investigated to include other artificial intelligent control methods such as neural control, self-learning control for better system performance.

\section{CONFLICT OF INTEREST}

The authors confirm that this article content has no conflict of interest.

\section{ACKNOWLEDGEMENTS}

This paper was funded by the project of Chinese International S \& T Cooperation (2011DFA10440).

\section{REFERENCES}

[1] C. V. Baysal, Erkmen, and M. Aydan, "An intelligent inference system for robot hand optimal grasp preshaping," International Journal of Computational Intelligence Systems, vol. 3, no. 5, pp. 656-673, 2010.

[2] Y. K. Cho, W. J. Hwang and W. S. Lee, "An intelligent control of robot hand with four fingers," In: Proceedings of $11^{\text {th }}$ International Conference on Control, Automation and Systems, Gyeonggi-do, 2011, pp. 1100-1104.

[3] C. H. Chen and D. Subbaram Naidu, "Hybrid control strategies for a five-finger robotic hand," Biomedical Signal Processing and control, vol. 8, no. 4, pp. 382-390, 2013.

[4] V. Lippiello, B. Siciliano, and L. Villani, "Multi-fingered grasp synthesis based on the object dynamic properties," Robotics and Autonomous Systems, vol. 61, no. 6, pp. 626-636, June 2013

[5] E. Mattar, "A survey of bio-inspired robotics hands implementation: New directions in dexterous manipulation," Robotics and Autonomous Systems, vol. 61, no. 5, pp. 517-544, 2013. 
[6] H. Wang, Y. Li, and Longxiao, "Realization of a hydraulic actuated biped robot," International Journal of Control, Automation, and Systems, vol. 12, no. 4, pp. 843-851, 2014.

[7] Y. Yang, and Q. X. Cao, "Monocular vision based 6D object localization for service robot's intelligent grasping," Computers \& Mathematics with Applications, vol. 64, no. 5, pp. 1235-1241, 2012.
[8] W. H. Li, and L. Kleeman, "Segmentation and modeling of visually symmetric objects by robot actions," International Journal of Robotics Research, vol. 30, no. 9, pp. 1124-1142, 2011.

[9] Z. Shang, Y. Li , X. Deng, and L. Li. "Slip sense sensor based on micro-vibration detection," Journal of Vibration and Shock, vol. 26, no. 12, pp. 135-137, 2007.

Received: September 16, 2014

Revised: December 23, 2014

Accepted: December 31, 2014

(C) Zhendong et al.; Licensee Bentham Open.

This is an open access article licensed under the terms of the (https://creativecommons.org/licenses/by/4.0/legalcode), which permits unrestricted, non-commercial use, distribution and reproduction in any medium, provided the work is properly cited. 